\title{
Localization of electrodes in the subthalamic nucleus on magnetic resonance imaging
}

\author{
Claudio Pollo, M.D., ${ }^{1,5}$ François Vingerhoets, M.D., ${ }^{2}$ Etienne Pralong, Ph.D., ${ }^{3}$ \\ Joseph Ghika, M.D., ${ }^{2}$ Philippe Maeder, M.D. ${ }^{4}$ Reto Meuli, Ph.D. ${ }^{4}$ \\ Jean-Philippe Thiran, Ph.D., ${ }^{5}$ and Jean-Guy Villemure, F.R.C.S.C. ${ }^{1}$
}

Departments of ${ }^{1}$ Neurosurgery, ${ }^{2}$ Neurology, and ${ }^{4}$ Radiology; ${ }^{3}$ Neurosurgical Neurophysiology Institute, Centre Hospitalier Universitaire Vaudois; and ${ }^{5}$ Signal Processing Institute, Ecole Polytechnique

Fédérale, Lausanne, Switzerland

\begin{abstract}
Object. The authors describe a new method of localizing electrodes on magnetic resonance (MR) images and focus on the positions of both the most efficient contact and the electrode related to the MR imaging target.

Methods. Thirty-one patients who had undergone bilateral subthalamic nucleus (STN) deep brain stimulation (DBS) were included in this study. Target coordinates were calculated in the anterior commissure-posterior commissure referential. A study of the correlation between the artifact and the related contact allowed one to deduce the contact position from the identification of the distal artifact on MR imaging. The best stimulation point corresponded with the contact resulting in the best Unified Parkinson's Disease Rating Scale (UPDRS) motor score improvement. It was compared (Student t-test) with the dorsal margin of the STN (DM STN), which was determined electrophysiologically. The distance between the target and the electrode was calculated individually in each axis.

The best stimulation point was located at anteroposterior $-2.34 \pm 1.63 \mathrm{~mm}$, lateral $12.04 \pm 1.62 \mathrm{~mm}$, and vertical $-2.57 \pm 1.68 \mathrm{~mm}$. This point was not significantly different from the DM STN $(\mathrm{p}<0.05)$. The postoperative UPDRS motor score was $28.07 \pm 12.16$, as opposed to the preoperative score of $46.27 \pm 13.89$. The distance between the expected and actual target in the $\mathrm{x}$ - and $\mathrm{y}$-axes was $1.34 \pm 1.02$ and $1.03 \pm 0.76 \mathrm{~mm}$, respectively. In the $\mathrm{z}$-axis, $39.7 \%$ of the distal contacts were located proximal to the target.

Conclusions. This approach proposed for the localization of the electrodes on MR imaging shows that DBS is most effective in the dorsal and lateral part of the STN and indicates that the DBS electrode can be located more proximally than originally expected because of the caudal brain shift that may occur during the implantation procedure.
\end{abstract}

KeY Words • Parkinson disease • subthalamic nucleus • deep brain stimulation • magnetic resonance imaging • electrode

$\mathrm{H}$ IGH-FREQUENCY electrical stimulation of the STN has been shown to be a highly efficient therapy for advanced PD. ${ }^{11,12,21}$ According to the classic pathophysiological model, ${ }^{2,5}$ which is supported by electrophysiological data from animal studies, the net effect of DBS should result from the inhibition of STN excitatory output on the globus pallidus internus and SN. Precision in reaching the target is therefore mandatory to obtain optimal clinical improvement. ${ }^{3,4}$ However, some controversies exist about the optimal site of stimulation and the local mechanisms of action of STN DBS. ${ }^{6,8,22,25}$ More accurate understanding of the effect(s) of DBS implies perfect knowledge of the actual postoperative position of the implanted electrodes and active contact. Magnetic resonance imaging has

\footnotetext{
Abbreviations used in this paper: $\mathrm{AC}-\mathrm{PC}=$ anterior commissureposterior commissure; $\mathrm{AP}=$ anteroposterior; $\mathrm{CSF}=$ cerebrospinal fluid; DBS = deep brain stimulation; $\mathrm{LAT}=$ lateral; $\mathrm{MCP}=$ midcommissural point; MPRAGE = magnetization-prepared rapid acquisition gradient echo; $\mathrm{MR}=$ magnetic resonance; $\mathrm{PD}=$ Parkinson disease; $\mathrm{SN}=$ substantia nigra; $\mathrm{STN}=$ subthalamic nucleus; SWA $=$ Schaltenbrand and Wahren atlas; UPDRS = Unified PD Rating Scale; VERT = vertical.
}

inspired interest in postoperative control of the electrode localization. Authors of several studies based on MR imaging alone or multimodal imaging coregistration have described methods for electrode localization. . $^{15,17,18,26}$ In these studies, it has been assumed that the position of the electrode artifact and the actual electrode position are highly correlated, but the accuracy of direct localization of the artifact remains to be determined.

In the present study, we describe a new procedure based on the knowledge of the relationship between the artifact and related contact, which allows one to localize each contact of the electrode and focus on the position of the stimulated contact in the AC-PC referential in correlation with the electrophysiological position of the dorsal margin of the STN as well as the position of the electrode related to the preoperative target.

\section{Clinical Material and Methods}

Patient Selection, Surgical Planning, and Implantation Procedure

Thirty-one patients (18 males and 13 females, mean age $63 \pm 8$ years) with advanced PD treated with bilateral STN 
DBS surgery at the Lausanne University Hospital were included in this study. For our purposes, we selected only patients who received implants after one microrecording and one macrostimulation trajectory and were permanently stimulated on one contact on a monopolar basis. Surgical planning was performed using a stereotactic $3 \mathrm{D} \mathrm{T}_{1}$-weighted MPRAGE MR imaging sequence (Vision, 1.5 tesla; Siemens) for identification of the $\mathrm{AC}$ and $\mathrm{PC}$ and the MCP. The target was then selected on a 3-mm-thick inversion recovery $\mathrm{T}_{2}$-weighted coronal slice obtained perpendicular to the AC-PC line and crossing the anterior pole of the red nucleus, that is, generally $3 \mathrm{~mm}$ posterior to the MCP, and placed in the inferolateral portion of the subthalamic zone, limited superiorly by the thalamus, laterally by the pyramidal tract, inferiorly by the SN, and medially by the midline $;^{13}$ this position corresponds to the inferior portion of the STN according to the SWA. ${ }^{16}$ Final electrodes were implanted while the patient was in a state of local anesthesia following electrophysiological and clinical mapping of the STN. A 3-mm twist drill hole was made, and the dura mater was opened using a coagulation probe that did not exceed the diameter of our guiding tubes to prevent important CSF leakage and to allow for the insertion of microrecording (Medtronic) and macrostimulation electrodes. Extracellular single-unit recordings were performed starting at $-8 \mathrm{~mm}$ of the MR imaging-calculated target by using a stainless-steel microelectrode with a tip diameter shorter than $1 \mu \mathrm{m}$ and an impedance of $1 \pm 0.2 \mathrm{M} \Omega$ at $1 \mathrm{kHz}$ (Medtronic), which was mounted on a sliding canula. The electrode was advanced in $10-\mu \mathrm{m}$ steps using a manual hydraulic microdrive (model 51421; Stoelting). The indifferent electrode was connected to the sliding canula guide and the stereotactic frame. Signals from the microelectrode and the indifferent electrode were fed to a Leadpoint data recording and analysis station (Medtronic) in which it was amplified (total gain 200) and filtered (bandwidth $300-10000 \mathrm{~Hz}$, notch filter at $50 \mathrm{~Hz}$ ). The single unit discharges were visualized on a computer screen and fed into an audio monitor. During recording, based on signal background level (neuronal noise) and cell spiking frequency, the neurophysiologist described the successive typical recording steps, comprising the thalamus, the Forel fields/zona incerta, the STN, and eventually the SN. Thalamic recordings consisted of a heterogeneous population of bursting, pausing, regular spiking, kinesthetic, or tremor-locked neurons, with a mean frequency of $24.8 \pm$ $1.4 \mathrm{~Hz}$. These were followed by a silent zone $(1-2 \mathrm{~mm})$ with occasional isolated regular firing cells corresponding to the fields of Forel fibers/zona incerta neurons. The STN neurons discharged with a significantly higher spiking frequency $(42.3 \pm 1.8 \mathrm{~Hz})$, and a higher neuronal noise was observed. (At the bottom of the STN, SN neurons were regular spiking neurons often locked with heart pulsation).

The clinical effects of electrical stimulation on rigidity and/or tremor as well as the adverse effects were carefully studied and documented by a neurologist. A macroelectrode (TCB A011; Leibinger) and a neurostimulator (Neuro N50, Leibinger) were used. The initial assessment was performed $6 \mathrm{~mm}$ proximal to the target at 2,50 , and $200 \mathrm{~Hz}$ and proceeded by steps of $2 \mathrm{~mm}$ until reaching the target or, in some cases, more distally until no more clinically positive effects were obtained. The definitive electrode (Activa 3389; Medtronic) was implanted under fluoroscopic control. The distal contact of the electrode was used as the ref- erence point and was intentionally placed 0 to $2 \mathrm{~mm}$ distal to the target or the more distal point at which clinical benefits were obtained to optimally cover the region of interest, and the final electrode was finally secured behind the bur hole with a titanium miniplate.

\section{Target Localization in the AC-PC Referential}

On the selected inversion recovery $\mathrm{T}_{2}$-weighted MR imaging slice, the $2 \mathrm{D}$ coordinates of the nine $\mathrm{CRW}$ frame rods and the STN targets were determined. The entire data set was treated using the UCLF software (Radionics), which calculated the AP (x), LAT (y), and VERT (z) coordinates of each target in the referential of the stereotactic frame (frame referential). Given that the SWA coordinates were related to the $\mathrm{AC}-\mathrm{PC}$ referential with the $\mathrm{MCP}$ as the origin, translation and rotation matrices were defined to transpose the target coordinates from the frame into the AC-PC referential (Appendix 1).

\section{Electrode Localization in the AC-PC Referential}

A postoperative $3 \mathrm{D} \mathrm{T}_{1}$-weighted MPRAGE MR imaging sequence was performed and defined in the AC-PC referential. The distal limit of the artifact was found to be the most reliable for identification, considering the significant overlapping between the artifacts surrounding the intermediate contacts and the artifact induced by the connecting cables immediately proximal to the proximal contact artifact. As the electrode reaches the target through a double oblique (lateromedial and AP) approach, the distal artifact was more precisely identified on sagittal and coronal slices, following the main axis of the electrode. The projected angle of the electrode with the VERT on the coronal and sagittal planes was also measured (Fig. 1). Data from an in vitro and in vivo study ${ }^{14}$ on the electrode artifacts confirmed that both contact and corresponding artifacts have the same center, whereas the distal tip of the artifact and the center of the distal contact are separated by $2.15 \mathrm{~mm}$ (Fig. 2). Thus, an algorithm based on trigonometric and Pythagorean relations was developed according to the equations described in $A p$ pendix 2 to calculate the coordinates of the center of each contact (distal to proximal: $\mathrm{C} 0, \mathrm{C} 1, \mathrm{C} 2$, and $\mathrm{C} 3$ ), knowing the height of one contact $(1.5 \mathrm{~mm})$ and the distance between two adjacent contacts $(0.5 \mathrm{~mm})$,

\section{Electrophysiological Localization of the STN and Determination of the Best Motor Stimulation Point}

The coordinates of the dorsal margin of the STN were obtained by calculating the AP and LAT coordinates of the implanted electrode at the VERT level of the implantation trajectory (related to the target) corresponding to the most proximal point at which the typical pattern of the STN firing neurons was observed. We excluded 13 cases in which recordings were not performed with the same microelectrode and the same analysis methods.

In the postoperative phase, the clinical effect of stimulation was evaluated for all contacts by the neurologists. The contact producing the greatest reduction in the UPDRS motor scale score on the follow-up evaluation without impairing side effects was considered as the best motor stimulation point. The duration of the permanently stimulated contact was calculated at the time of the study. 


\section{Pollo et al.}

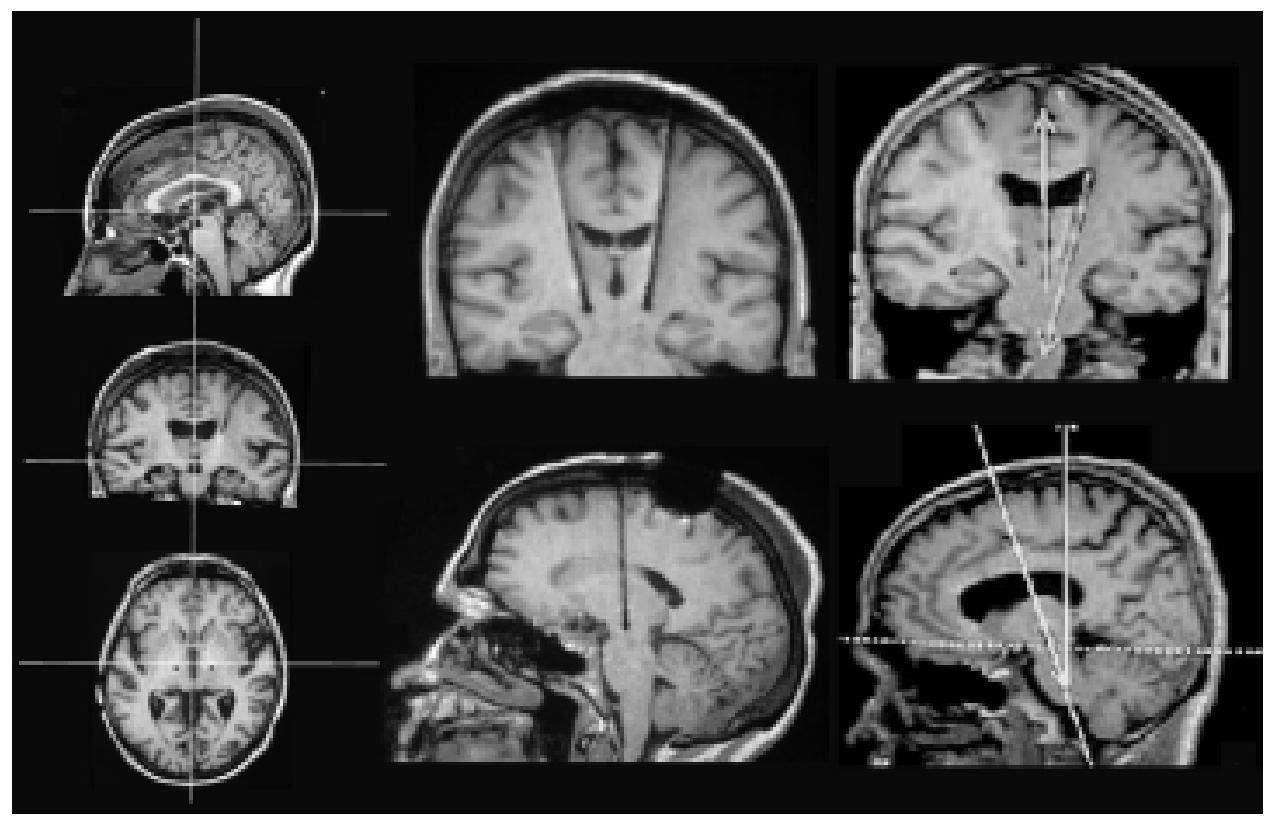

FIG. 1. Magnetic resonance images demonstrating the procedure to localize the implanted electrodes. Left: Defining the brain volume in the AC-PC referential with the MCP as the origin (white crosses). Center: Identifying the limits of the distal artifact on coronal (upper) and sagittal (lower) slices, following the main axis of the electrode. Right: Measuring the projected angles between the electrode and the VERT plane in coronal (upper) and sagittal (lower) planes.

A bilateral Student t-test $(\alpha=0.025)$ was performed to compare the AP, LAT, and VERT coordinates of the electrophysiologically determined dorsal margin of the STN and the clinically assessed best motor stimulation point.

\section{Distance Between the Preoperative Target and Implanted Electrode}

Following our protocol, the distal limit of $\mathrm{C} 0$ is intentionally placed 0 to $2 \mathrm{~mm}$ distal to the target in almost all of
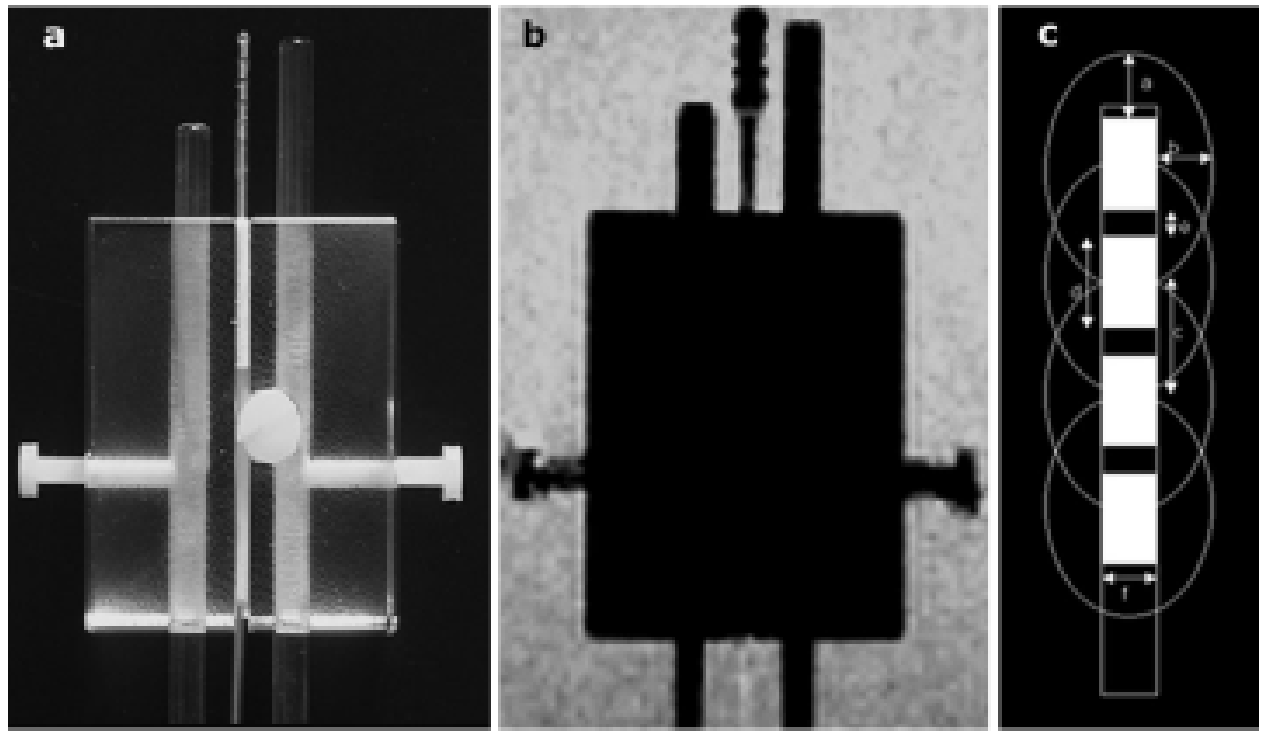

FIG. 2. a: Photograph depicting the Plexiglas referential receiving in the same plane the electrode between two parallel sliding cylindrical localizers placed at the distal limit of $\mathrm{C} 0$ and the proximal limit of $\mathrm{C} 3$. b: Magnetic resonance imaging reconstruction revealing the electrode and the referential from a 3D $\mathrm{T}_{1}$-weighted MPRAGE acquisition. An artifact is identifiable on each contact. The limits and the distal artifact are the most reliable. c: Illustration showing the dimensions and overlapping artifacts induced by the stimulating contacts of the electrode. Labels: $a=1.4 \mathrm{~mm} ; \mathrm{b}=1.16 \mathrm{~mm} ; \mathrm{c}=2.3$ $\mathrm{mm} ; \mathrm{d}=1.5 \mathrm{~mm} ; \mathrm{e}=0.5 \mathrm{~mm} ; \mathrm{f}=1.27 \mathrm{~mm}$. 
our cases and therefore cannot be directly compared with the original target, because it is expected to be deeper, more medial, and posterior. Instead, to study the AP and LAT distances between the preoperative target and the electrode, we used the actual coordinates of the electrode at the point at which it reached the VERT coordinate of the MR imaging target. When the distal tip of the electrode was found more proximal to the target, the electrode trajectory was virtually prolonged until the level of the VERT coordinate of the MR imaging target. The AP and LAT distances were obtained considering the absolute value of the difference between coordinates of the target and electrode $\left(\left|A P_{\text {tareet }}-A P_{\text {electrode }}\right|\right.$ and $\left|L A T_{\text {target }}-L A T_{\text {electrode }}\right|$, respectively). For direction considerations, positive values indicate that the preoperative target is more anterior and LAT than the electrode. The AP and LAT distances were distributed in intervals of $1 \mathrm{~mm}$ beginning from $0(0-1 \mathrm{~mm}, 1-2 \mathrm{~mm}$, and so forth).

The VERT distance between the target and the distal limit of $\mathrm{C} 0$ were studied separately. We considered positive (electrode more distal than target) and negative (target more distal than electrode) values to calculate the VERT distance.

\section{Results}

The coordinates of the target, the electrode contacts, the best stimulation points, and the dorsal margin of the STN are related to the AC-PC referential and were projected on selected slices of the SWA: ${ }^{16}$ the coronal slice was taken 3 $\mathrm{mm}$ posterior, the axial slice $4 \mathrm{~mm}$ inferior, and the sagittal slice $12 \mathrm{~mm}$ LAT to the MCP, which was used as the origin. The coordinates are expressed in millimeters. A negative AP value indicates a target projecting behind the MCP, a negative VERT value indicates a target projecting below the MCP, and a negative LAT value indicates a target located on the left side.

\section{Position of the Target}

The coordinates of $62 \mathrm{MR}$ imaging-defined targets were obtained. The mean target was found with the following coordinates: $\mathrm{AP}=-2.78 \pm 1.38 \mathrm{~mm}, \mathrm{LAT}=12.04 \pm 1.20$ $\mathrm{mm}$, and VERT $=-5.89 \pm 1.63 \mathrm{~mm}$ and illustrated in Fig. 3.

\section{Position of the Electrode Contacts}

The positions of 248 electrode contacts were calculated. The mean coordinates (and standard deviations) were as follows (in millimeters): $\mathrm{C} 0, \mathrm{AP}=-3.49 \pm 1.51$, LAT $=$ $11.36 \pm 1.30), \mathrm{VERT}=-5.37 \pm 1.72 ; \mathrm{C} 1, \mathrm{AP}=-2.79 \pm$ $1.54, \mathrm{LAT}=11.73 \pm 1.32$, VERT $=-3.56 \pm 1.73$; $\mathrm{C} 2, \mathrm{AP}=-2.09 \pm 1.53, \mathrm{LAT}=12.10 \pm 1.36$, VERT $=$ $-1.74 \pm 1.74 ; \mathrm{C} 3, \mathrm{AP}=-1.39 \pm 1.54, \mathrm{LAT}=12.47 \pm$ $1.40, \mathrm{VERT}=0.06 \pm 1.75$. These points are also illustrated in Fig. 3.

\section{Dorsal Margin of the STN, the Best Stimulation Point, and UPDRS Scores}

The locations of 36 electrophysiologically determined positions of the dorsal margin of the STN were calculated and are summarized as follows: $\mathrm{AP}=-2.89 \pm 1.58$, $\mathrm{LAT}=12.51 \pm 1.42$ ), and VERT $=-2.66 \pm 1.34$ (Fig. 4).

The coordinates of 62 stimulated contacts were calcu- lated. The following coordinates for the best motor point were obtained (in millimeters): $\mathrm{AP}=-2.34 \pm 1.63$, LAT $=$ $12.04 \pm 1.62$, VERT $=-2.57 \pm 1.68$ (Fig. 4). There was no significant difference $(\mathrm{p}<0.05)$ in the location of the dorsal margin of the STN and the position of the best stimulation motor point.

The patients have continuously received stimulation through the contact producing the greatest reduction in the UPDRS motor scale score from 14 to 42 months (mean 26.3 months). The preoperative UPDRS III motor score without medication was $46.27 \pm 13.89$. Postoperatively, the score was reduced to $28.07 \pm 12.16$ under stimulation conditions.

\section{Distance Between the Preoperative Target and Implanted Electrode}

The AP and LAT distances between the target and implanted electrode are represented on Fig. 5. The mean AP distance was $1.34 \pm 1.02 \mathrm{~mm}$ and the mean LAT distance was $1.03 \pm 0.76 \mathrm{~mm}$. Forty-two $(67.7 \%)$ of 62 electrodes were found posterior to the target; and $40(64.5 \%)$ of 62 , LAT to the target. Regarding the AP distances, 27 (43.5\%) of 62 electrodes were $\leq 1 \mathrm{~mm}$ and $49(79 \%)$ of 62 were $\leq$ $2 \mathrm{~mm}$. Concerning the LAT distances, $37(59.7 \%)$ of 62 electrodes were $<1 \mathrm{~mm}$ and $55(88.7 \%)$ of 62 were $<2$ mm (Fig. 5). We obtained a VERT distance of $0.21 \mathrm{~mm}$. Distal contacts were found proximal to the target in 28 $(46.7 \%)$ of 62 cases.

\section{Discussion}

\section{Accuracy in Localizing Implanted Electrodes on MR Imaging}

Several MR imaging-based approaches to localize implanted electrodes have already been described using MR imaging alone, ${ }^{15,18}$ atlas-MR imaging, ${ }^{26}$ or multimodality imaging coregistration. ${ }^{17}$ In these studies, it was assumed that the artifact and related contact were congruent, but the reliability of performing electrode localization based on direct identification of the artifact is not precisely known. Moreover, the accuracy of the coregistration algorithms used for the purpose of electrode localization has not been extensively analyzed. We have previously shown that the artifact is circular in an image plane perpendicular to the electrode axis and that this artifact is perfectly centered on the contact. Furthermore, the dimensions and shape of the artifact were not significantly modified according to the frequency-encoded gradient and phase directions. However, we found that there was a significant overlap between adjacent artifacts and that the presence of the artifact induced by the thin connecting cables, which were identifiable all along the axis of the electrode, might have reduced the precision in localization of the three proximal contacts by direct identification of the related artifacts. Given that the identification of the limits of the distal artifacts is most reliable on MR imaging and knowing the distances between them and the actual center of the distal contact, our indirect approach allows precise localization of each contact by using Pythagorean and trigonometric relations. The entire process can be performed using an algorithm similar to the one proposed in Appendix 2 (Fig. 6). With this simple approach, imprecision in identifying the distal limits of the $\mathrm{C} 0$ artifact can result 
C. Pollo et al.
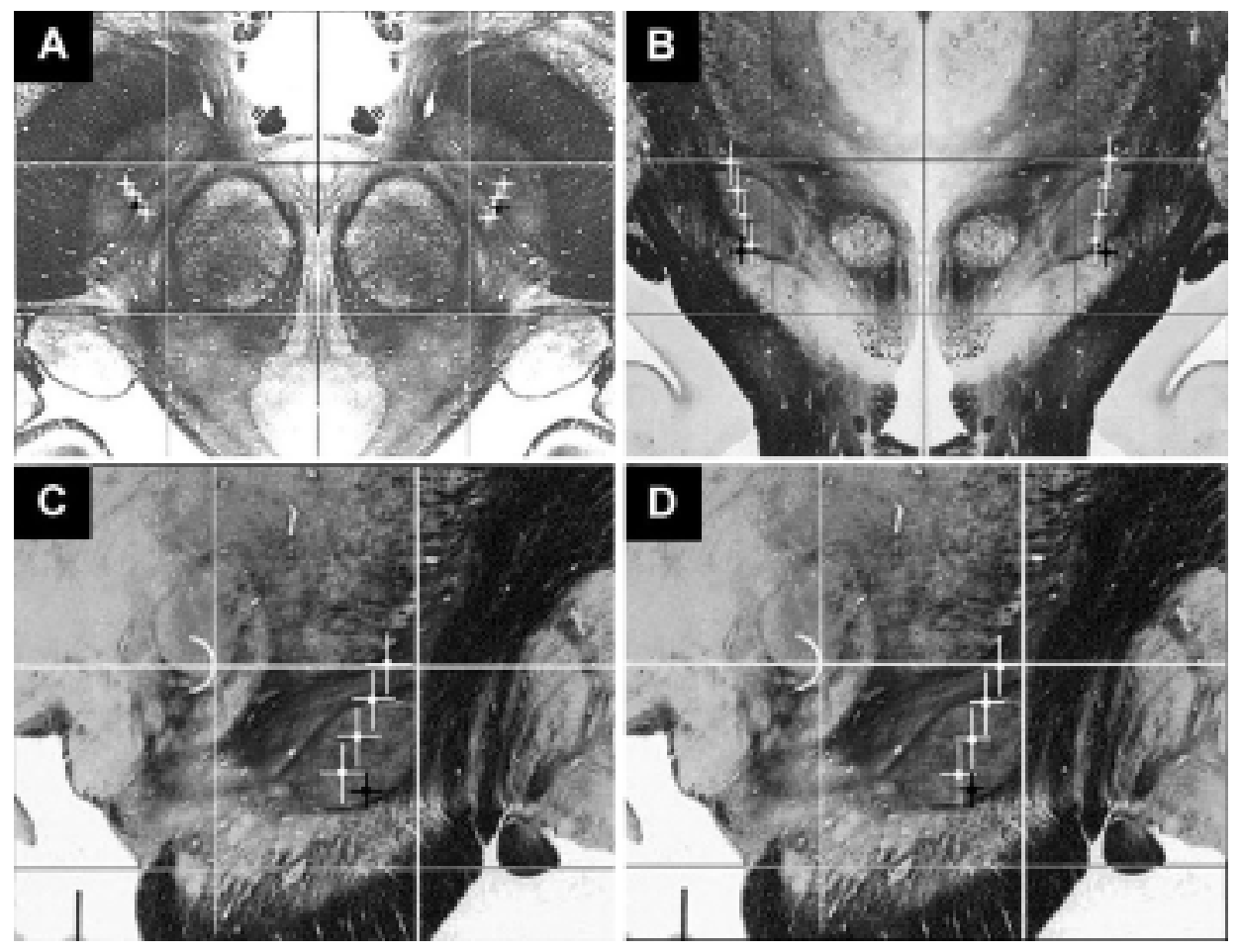

FIG. 3. Projections demonstrating the mean preoperative targets (black circles with crosses) and the mean four electrode leads (white circles with crosses) on images from the SWA. A: Axial section obtained $4 \mathrm{~mm}$ below the MCP. B: Coronal section obtained $3 \mathrm{~mm}$ posterior to the MCP. C and D: Right (C) and left (D) sagittal sections obtained $12 \mathrm{~mm}$ LAT to the MCP. Circles represent the mean coordinates; crosses, the standard errors. Reprinted with permission from Schaltenbrand G, Wahren W: Atlas for Stereotaxy of the Human Brain, ed 2. Stuttgart: Thieme, 1977.
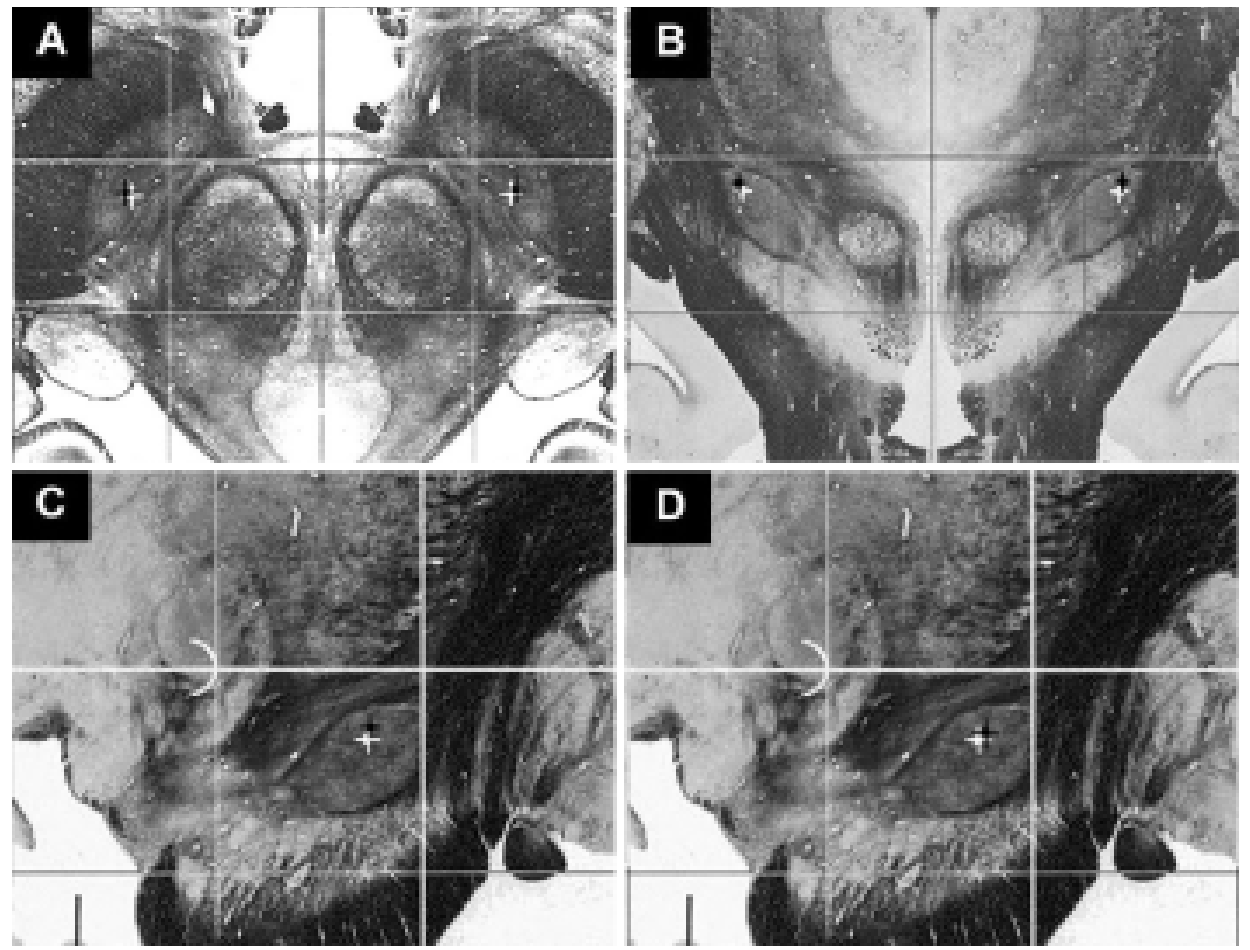

FIG. 4. Projections revealing the best motor stimulation point (white circles with crosses) and the dorsal limit of the STN as determined electrophysiologically (black circles with crosses) on the SWA. A: Axial section obtained $4 \mathrm{~mm}$ below the MCP. B: Coronal section obtained $3 \mathrm{~mm}$ posterior to the MCP. C and D: Right (C) and left (D) sagittal sections obtained $12 \mathrm{~mm}$ LAT to the MCP. Circles represent the mean coordinates; crosses, the standard errors. Reprinted with permission from Schaltenbrand G, Wahren W: Atlas for Stereotaxy of the Human Brain, ed 2. Stuttgart: Thieme, 1977. 
from the voxel size of the MR gradient echo acquisition $(1.09 \times 1.09 \times 1.25 \mathrm{~mm})$. The high 3D spatial resolution of the 3D MPRAGE sequence provides a suitable method to localize the limits of $\mathrm{C} 0$ with a maximal error that should not exceed half the voxel size $(0.5 \times 0.5 \times 0.6 \mathrm{~mm})$.

Similarly, imprecise identification of the projected angles of the electrode on coronal and sagittal planes can lead to increasing errors from $\mathrm{C} 0$ to $\mathrm{C} 3$. An overestimation of $5^{\circ}$ in these two angles would induce on $\mathrm{C} 3$ an anterior and LAT shift of approximately $0.6 \mathrm{~mm}$. Finally, errors resulting from image distortion on MR imaging for the purpose of stereotactic procedures have already been evaluated using high field gradient echo sequences ${ }^{19}$ and were found inferior to $1 \mathrm{~mm}$ in each of the AP, LAT, and VERT axes.

\section{Electrode Localization and the Best Stimulation Motor Point}

According to the SWA, C0 projects into the inferior portion of the STN. Considering the double oblique trajectory of the electrode, $\mathrm{C} 1, \mathrm{C} 2$, and $\mathrm{C} 3$ should be located cranially, anteriorly, and laterally to C0. As shown in Fig. 2, C1 and $\mathrm{C} 2$ are located inside the STN, whereas $\mathrm{C} 3$ projects above the STN in the Forel fields/zona incerta region. Although transferring the electrode coordinates in the ACPC referential onto sections of the SWA does not provide any normalization procedure to correct for atlas-patient brain size variations, it does prevent errors that can result from multimodality image or image-atlas coregistration methods that are difficult to evaluate at a voxel level for a defined brain area. ${ }^{7}$

Applying our localization technique, we found that the point producing the best effects on motor symptoms of PD was centered in the dorsal and LAT part of the STN (obtained in 31 patients) and correlated with the position of the dorsal margin of the STN, which was determined electrophysiologically (recordings from 18 patients). Results of previous electrophysiological studies have shown that movement-related cells in the human STN (sensorimotor part) were located in the dorsal part of the nucleus, ${ }^{1,20}$ suggesting that the sensorimotor part of the STN should be the best point for electrical stimulation with respect to the current pathophysiological model of PD. ${ }^{2,5}$ Data in recent publications have shown that the most effective stimulation point on parkinsonian motor symptoms was located in the dorsal and LAT portion of the STN.$^{10,18}$ However, we found that some of the best stimulation points projected proximal to the anatomical limits of the STN (Fig. 6). This observation agrees with previous study data ${ }^{8,9,22,23,26}$ indicating that other possible structures or fibers (output pallidothalamic tracts of the Forel fields and zona incerta) can be involved in STN DBS and addressing the question of the local mechanism of action of high-frequency electrical stimulation (activation rather than inhibition or both).

\section{Distance Between the Preoperative Target and Implanted Electrode}

We found that in most cases the distal contact of the electrode was placed $\leq 2 \mathrm{~mm}$ from the preoperative target in the AP and LAT axes (79 and $88.7 \%$, respectively). The slightly greater imprecision in the AP axis can be explained by the frequency encoding direction that contributes to a
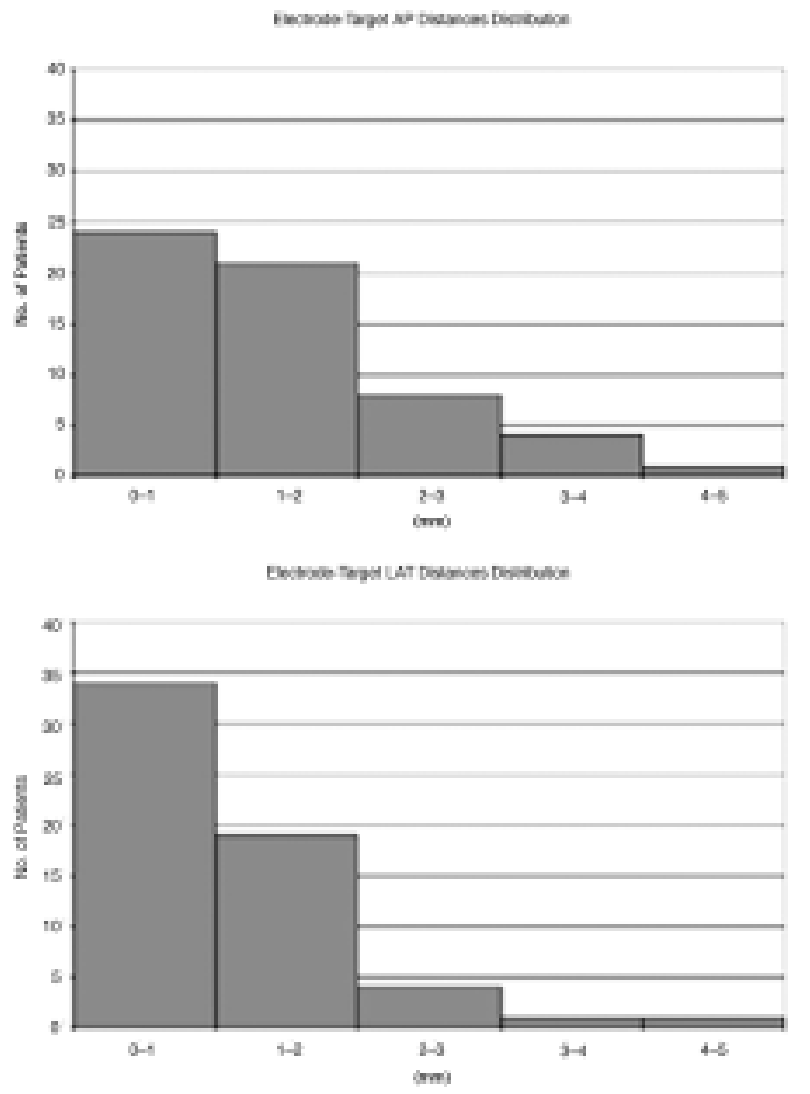

FIG. 5. Graphs demonstrating the distribution of the distances between the preoperative target and the electrode at the VERT coordinate of the target. Upper: Anteroposterior. Lower: Lateral.

higher distortion in this axis. Surprisingly, the distal tip of the electrode was located proximal to the preoperative target in $46.7 \%$ of cases, whereas it was expected to be 0 to 2 $\mathrm{mm}$ distal to that point in all cases.

A possible cause of this discrepancy is intraoperative brain shift that can result from intraoperative CSF leakage; displacement of the entire brain within the skull while the patient is placed in the semisitting position in the operating room whereas MR acquisition is performed with the patient supine; and displacement of the brain caused by the resistance of the cerebral gray and white matter against the penetration of the macroelectrodes and guide tubes. All these phenomena certainly contribute to a caudal displacement of the brain and/or a narrowing of the third ventricle and interpeduncular cistern, which can result in displacement of the target in the caudal and/or medial directions. The amount of intraoperative CSF leakage is difficult to evaluate, although it may be significant, especially in bilateral procedures. For this reason, we prefer to open the dura by coagulation not exceeding the diameter of our guide tubes. Brain displacement between the supine and semisitting positions can be expected in parkinsonian patients prone to brain atrophy as part of their disease and as a consequence of an "aging" phenomenon (patients who undergo surgery are generally older than 60 years of age). This circumstance can be prevented by operating on supine patients. Note, however, that the supine position can increase intraoperative CSF leakage.

Our findings regarding the discrepancy between the ex- 
C. Pollo et al.
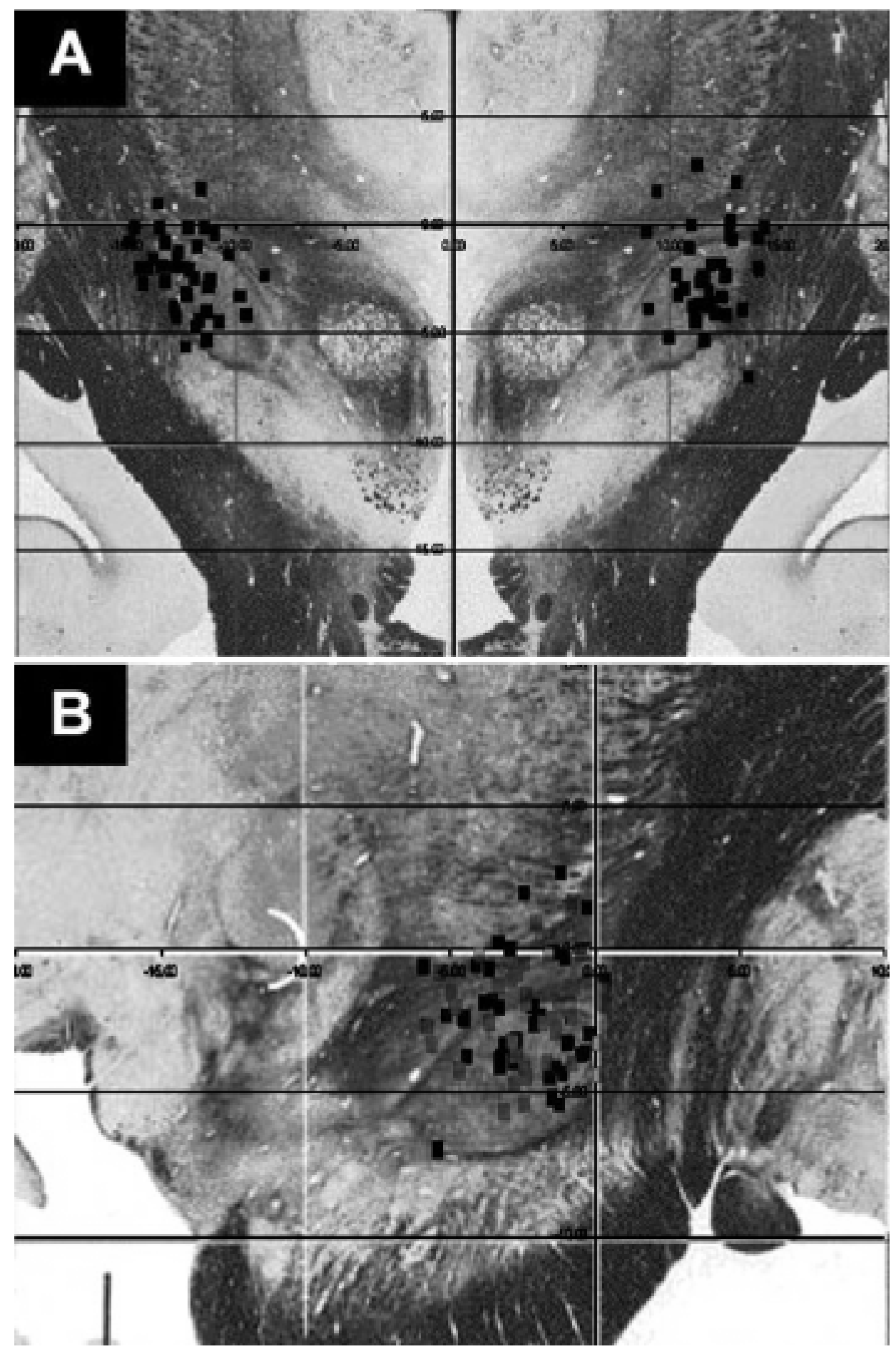

FIG. 6. Projections exhibiting the best stimulation points. A: Coronal section obtained $3 \mathrm{~mm}$ posterior to the MCP. B: Right (gray squares) and left (black squares) sagittal sections obtained $12 \mathrm{~mm}$ LAT to the MCP. Reprinted with permission from Schaltenbrand G, Wahren W: Atlas for Stereotaxy of the Human Brain, ed 2. Stuttgart: Thieme, 1977.

pected and observed distance in the VERT axis are in accordance with Wester and Krakenes' ${ }^{24}$ recent observations of a VERT displacement of the cerebral cortex from 0 to $9 \mathrm{~mm}$ in 11 patients who underwent thalamotomies while in the sitting position, thus necessitating a subsequent advance of the electrode from 1 to $5.5 \mathrm{~mm}$ (mean $3.5 \mathrm{~mm}$ ) to reach the target. They also reported a tendency toward medial displacement of the target, which could explain why electrodes were found LAT to the target in $64.5 \%$ of cases in the present study. We have clinical evidence that electrodes may tend to be LAT to the target. In patients in whom the electrode had to be moved intraoperatively from the anatomical 

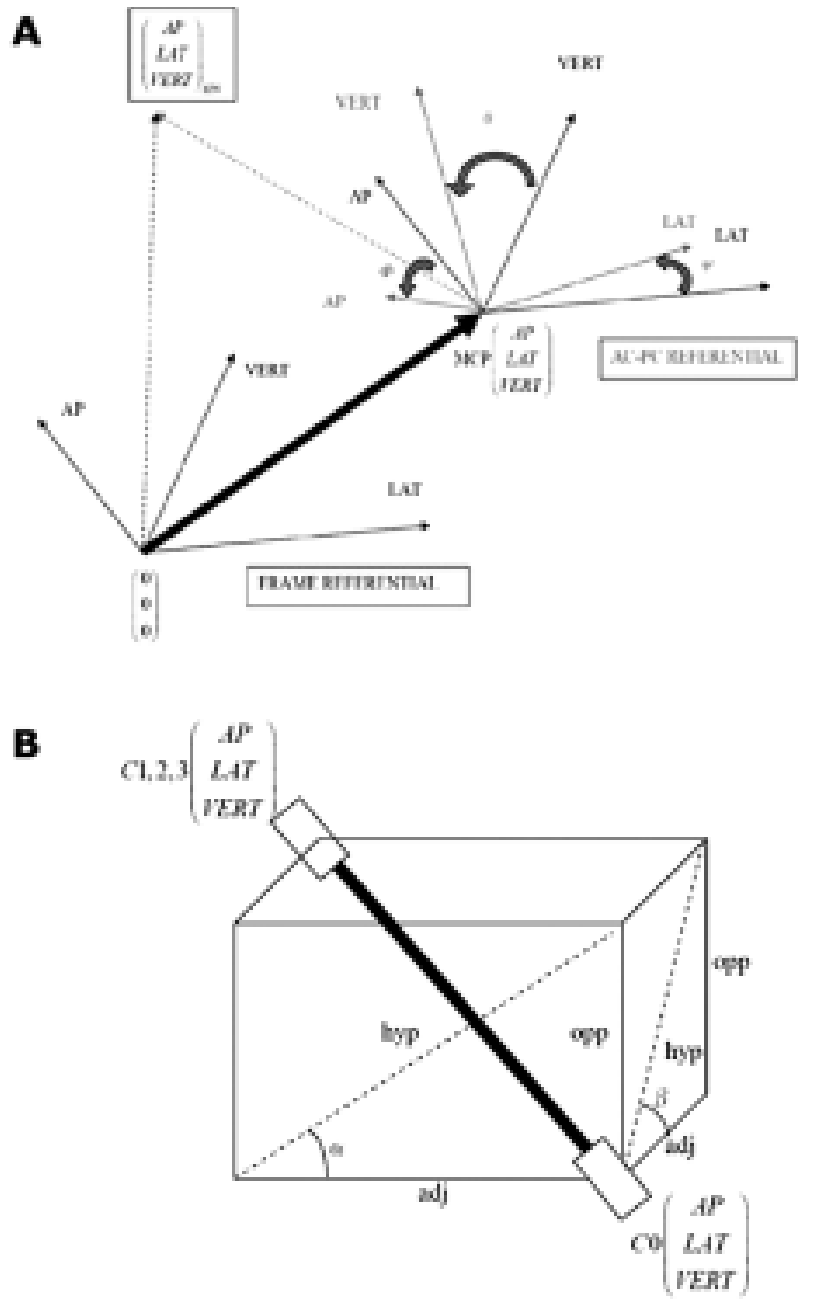

FIG. 7. A: Schematic featuring the transformation of the STN coordinates from the frame into the AC-PC referential using translation and rotation matrices. B: Schematic showing the calculation of the coordinates of the center of each contact starting from $\mathrm{C} 0$ using Pythagorean and trigonometric laws.

target $(12.75 \%)$, based on the clinical response and adverse effects of macrostimulation as well as microelectrode recordings, displacement occurred in a medial direction in $75 \%$ of cases, whereas $16.6 \%$ showed LAT and $8.3 \%$ demonstrated anterior displacement. Although we have no clear explanation for the tendency of electrodes to be placed posterior to the target, we suppose that the VERT brain displacement occurring while the patient is in the semisitting position can be accompanied by a less remarkable anterior motion.

Further independent data are needed to verify our hypotheses, quantify the influence of each contributive factor, and minimize intraoperative brain displacement. Intraoperative brain shift could be more precisely studied with intraoperative imaging. However, available intraoperative MR imaging technologies still have low resolution and allow only partial imaging of the brain. Subsequent image processing tools are needed to predict brain displacement at distant sites or require compatible MR imaging stereotactic devices to prevent major distortions. Nevertheless, because our targeting method is performed in the inferior portion of the STN, we can expect that in cases of caudal brain shift, at least one electrode contact will be located inside the sensorimotor part of the STN. We also believe that information provided by microrecordings around the level of the ventral margin of the STN and precise assessment of the most distal level of clinical efficacy induced by intraoperative stimulation are helpful parameters to compensate for brain shift and to refine the final electrode position.

\section{Conclusions}

In the present study we proposed an approach for accurately localizing the implanted electrodes on postoperative volumetric MR imaging based on the identification of the distal artifact, the measurement of the projected electrode angles in the sagittal and coronal planes, and the use of Pythagorean and trigonometric relations. The electrode position was correlated with the preoperative target, except in the z-axis, where the electrode was found proximal to the expected location. This outcome can be explained mainly by the caudal brain displacement occurring while the patient is in the semisitting position or provoked by the guide tubes and/or electrode penetration and by the intraoperative CSF leakage.

This observation should be taken into consideration during the surgical procedure to prevent misplacement of definitive electrodes. According to the SWA, STN DBS appears to be most effective in the dorsal and LAT part of the STN and corresponds to the dorsal margin of the STN as determined electrophysiologically.

\section{Appendix 1}

Transformation of STN Coordinates From the Frame Referential to the AC-PC Referential

Translation. The origin of the frame referential must be translated on the origin of the AC-PC referential, that is, the MCP (Fig. 7). Thus, the STN coordinates in the AC-PC referential [AP LAT VERT 0$]_{\text {STN }(M C P)}$ are obtained by multiplying the STN coordinates in the frame referential [AP LAT VERT 0 $]_{\mathrm{STN}(\mathrm{fr})}$ and the translation matrix, where the components of the last column of the matrix are the coordinates of the MCP in the frame referential

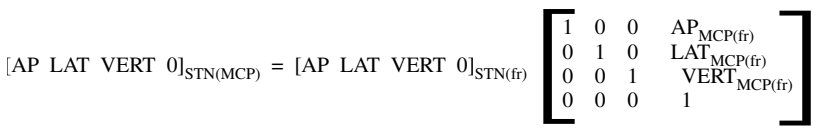

Rotation. According to the Euler rotation theorem, any rotation can be described using three angles. If the rotations are written in terms of rotation matrices $\mathrm{B}, \mathrm{C}$, and $\mathrm{D}$, then a general rotation $\mathrm{A}$ can be written as $\mathrm{A}=\mathrm{B} \times \mathrm{C} \times \mathrm{D}$, where

$\mathrm{B}=\left[\begin{array}{rrr}\cos \psi & \sin \psi & 0 \\ -\sin \psi & \cos \psi & 0 \\ 0 & 0 & 1\end{array}\right], \mathrm{C}=\left[\begin{array}{rrr}1 & 0 & 0 \\ 0 & \cos \theta & \sin \theta \\ 0 & -\sin \theta & \cos \theta\end{array}\right]$, and $\mathrm{D}=\left[\begin{array}{rrr}\cos \phi & \sin \phi & 0 \\ -\sin \phi & \cos \phi & 0 \\ 0 & 0 & 1\end{array}\right]$

Consequently, the STN coordinates in the AC-PC referential after rotation [AP LAT VERT $]_{\mathrm{STN}(\text { ar })}$ are obtained by multiplication of the STN coordinates in the AC-PC referential [AP LAT VERT 0] $]_{\mathrm{STN}(\mathrm{MCP})}$ with the matrix A

$$
\begin{aligned}
& {\left[\begin{array}{llll}
\text { AP } & \text { LAT } & \text { VERT }
\end{array}\right]_{\mathrm{STN}(\mathrm{ar})}=\left[\begin{array}{llll}
\text { AP } & \text { LAT } & \text { VERT } & 0
\end{array}\right]_{\mathrm{STN}(\mathrm{MCP})}} \\
& \times\left[\begin{array}{ccc}
\cos \psi \cos \phi-\cos \theta \sin \phi \sin \psi & \cos \psi \sin \phi+\cos \theta \cos \phi \sin \psi & \sin \psi \sin \theta \\
-\sin \psi \cos \phi-\cos \theta \sin \phi \cos \psi & -\sin \psi \sin \phi+\cos \theta \cos \phi \cos \psi & \cos \psi \sin \theta \\
\sin \theta \sin \phi & -\sin \theta \cos \phi & \cos \theta
\end{array}\right]
\end{aligned}
$$


where $\phi$ is the rotational angle about the $\mathrm{z}$-axis (angle between AC$\mathrm{PC}$ line and frame rods in the axial plane), $\theta$ is the rotational angle about the $\mathrm{X}$-axis (angle between AC-PC line and frame rods in the sagittal plane), and $\psi$ is the rotational angle about the z-axis again (angle between AC-PC line and frame rods in the coronal plane).

\section{Appendix 2}

Starting from the following general trigonometric and Pythagorean laws concerning right triangles

$\tan \alpha($ or $\beta)=$ opp/adj

$(\operatorname{adj})^{2}+(o p p)^{2}=(\text { hyp })^{2}$

and knowing the coordinates (AP, LAT, and VERT) of the distal limit of the distal artifact ( $\mathrm{C} 0$ artifact), the distance between the distal limit of the $\mathrm{C} 0$ artifact and the center of $\mathrm{C} 0(2.15 \mathrm{~mm})$, the projected angles of the electrodes in the coronal $(\alpha)$ and sagittal $(\beta)$ planes, the height of one contact $(1.5 \mathrm{~mm})$, we are able to deduce the coordinates of the center of $\mathrm{C} 0$. Knowing also the distance between two adjacent contacts $(0.5 \mathrm{~mm})$, we are able to deduce the coordinates of the centers of $\mathrm{C} 1, \mathrm{C} 2$, and $\mathrm{C} 3$ according to the following equations

$\mathrm{AP}_{(\mathrm{C} 0,1,2,3)}=\left(\tan \alpha \cdot \Delta_{(\mathrm{C} 0,1,2,3)}\right)+\left(\mathrm{AP}_{(\mathrm{C} 0,1,2,3)}-\mathrm{AP}_{(\mathrm{MCP})}\right)$

$\operatorname{LAT}_{(\mathrm{C} 0,1,2,3)}=\left(\tan \beta \cdot \Delta_{(\mathrm{C} 0,1,2,3)}\right)+\left(\mathrm{LAT}_{(\mathrm{C} 0,1,2,3)}-\mathrm{LAT}_{(\mathrm{MCP})}\right)$

$\operatorname{VERT}_{(\mathrm{C} 0,1,2,3)}=\Delta_{(\mathrm{C} 0,1,2,3)}+\left(\operatorname{VERT}_{(\mathrm{C} 0,1,2,3)}-\operatorname{VERT}_{(\mathrm{MCP})}\right)$

where

$\Delta_{(\mathrm{C} 0,1,2,3)}=\sqrt{\frac{(2.15 \text { or } 4.15 \text { or } 6.15 \text { or } 8.15)^{2}}{\left(1+\tan ^{2} \alpha\right)+\left(1+\tan ^{2} \beta\right)}}$

depending on the contact (use 2.15 for $\mathrm{C} 0,4.15$ for $\mathrm{C} 1$, and so forth). adj = adjacent side of the right angle; hyp = hypotenuse of the right triangle; opp $=$ opposite side of the right angle.

\section{References}

1. Abosch A, Hutchison WD, Saint-Cyr JA, Dostrovsky JO, Lozano AM: Movement-related neurons of the subthalamic nucleus in patients with Parkinson disease. J Neurosurg 97:1167-1172, 2002

2. Alexander GE, Crutcher MD: Functional architecture of basal ganglia circuits: neural substrates of parallel processing. Trends Neurosci 13:266-271, 1991

3. Benabid AL, Benazzous A, Pollak P: Mechanisms of deep brain stimulation. Mov Disord 17 (Suppl 3):S73-S74, 2002

4. Benazzouz A, Gao DM, Ni ZG, Piallat B, Bouali-Benazzouz R, Benabid AL: Effect of high-frequency stimulation of the subthalamic nucleus on the neuronal activities of the substantia nigra pars reticulata and ventrolateral nucleus of the thalamus in the rat. Neuroscience 99:289-295, 2000

5. DeLong MR, Alexander GE, Mitchell SJ, Richardson RT: The contribution of basal ganglia to limb control. Prog Brain Res 64: 161-174, 1986

6. Dostrovsky JO, Lozano AM: Mechanisms of deep brain stimulation. Mov Disord 17 (Suppl 3):S63-S68, 2002

7. Fitzpatrick JM, Hill DL, Shyr Y, West J, Studholme C, Maurer CR Jr: Visual assessment of the accuracy of retrospective registration of MR and CT images of the brain. IEEE Trans Med Imaging 17:571-585, 1998

8. Garcia L, Audin J, D'Alessandro G, Bioulac B, Hammond C: Dual effect of high-frequency stimulation on subthalamic neuron activity. J Neurosci 23:8743-8751, 2003

9. Hamel W, Fietzek U, Morsnowski A, Schrader B, Herzog J, Weinert D, et al: Deep brain stimulation of the subthalamic nucleus in Parkinson's disease: evaluation of active electrode contacts. J Neurol Neurosurg Psychiatry 74:1036-1046, 2003

10. Herzog J, Fietzek U, Hamel W, Morsnowski A, Steigerwald F, Schrader B, et al: Most effective stimulation site in subthalamic deep brain stimulation for Parkinson's disease. Mov Disord 19: 1050-1054, 2004

11. Kumar R, Lozano AM, Kim YJ, Hutchison WD, Sime E, Halket E, et al: Double-blind evaluation of subthalamic nucleus deep brain stimulation in advanced Parkinson's disease. Neurology 51: 850-855, 1998

12. Limousin P, Krack P, Pollak P, Benazzouz A, Ardouin C, Hoffmann D, et al: Electrical stimulation of the subthalamic nucleus in advanced Parkinson's disease. N Engl J Med 339:1105-1111, 1998

13. Pollo C, Meuli R, Maeder P, Vingerhoets F, Ghika J, Villemure JG: Subthalamic nucleus deep brain stimulation for Parkinson's disease: magnetic resonance imaging targeting using visible anatomical landmarks. Stereotact Funct Neurosurg 80:76-81, 2003

14. Pollo C, Villemure JG, Vingerhoets F, Ghika J, Maeder P, Meuli $\mathrm{R}$ : Magnetic resonance artifact induced by the electrode Activa 3389: an in vitro and in vivo study. Acta Neurochir (Wien) 146: 161-164, 2004

15. Saint-Cyr JA, Hoque T, Pereira LC, Dostrovsky JO, Hutchison WD, Mikulis DJ, et al: Localization of clinically effective stimulating electrodes in the human subthalamic nucleus on magnetic resonance imaging. J Neurosurg 97:1152-1166, 2002

16. Schaltenbrand G, Wahren W: Atlas for Stereotaxy of the Human Brain, ed 2. Stuggart: Thieme, 1977

17. Schrader B, Hamel W, Weinert D, Mehdorn HM: Documentation of electrode localization. Mov Disord 17 (Suppl 3):S167-S174, 2002

18. Starr PA, Christine CW, Theodosopoulos PV, Lindsey N, Byrd D, Mosley A, et al: Implantation of deep brain stimulators into the subthalamic nucleus: technical approach and magnetic resonance imaging-verified lead locations. J Neurosurg 97:370-387, 2002

19. Suh JS, Jeong EK, Shin KH, Cho JH, Na JB, Kim DH, et al: Minimizing artifacts caused by metallic implants at MR imaging: experimental and clinical studies. AJR Am J Roentgenol 171: 1207-1213, 1998

20. Theodosopoulos PV, Marks WJ Jr, Christine C, Starr PA: Locations of movement-related cells in the human subthalamic nucleus in Parkinson's disease. Mov Disord 18:791-798, 2003

21. Vingerhoets F, Villemure JG, Temperli P, Pollo C, Pralong E, Ghika J: Subthalamic DBS replaces levodopa in Parkinson's disease: two-year follow-up. Neurology 58:396-401, 2002

22. Vitek JL: Mechanisms of deep brain stimulation: excitation or inhibition. Mov Disord 17 (Suppl 3):S69-S72, 2002

23. Voges J, Volkmann J, Allert N, Lehrke R, Koulousakis A, Freund $\mathrm{HJ}$, et al: Bilateral high-frequency stimulation in the subthalamic nucleus for the treatment of Parkinson disease: correlation of therapeutic effect with anatomical electrode position. J Neurosurg 96:269-279, 2002

24. Wester K, Krakenes J: Vertical displacement of the brain and the target area during open stereotaxic neurosurgery. Acta Neurochir (Wien) 143:603-606, 2001

25. Wu YR, Levy R, Ashby P, Tasker RR, Dostrovsky JO: Does stimulation of the GPi control dyskinesia by activating inhibitory axons? Mov Disord 16:208-216, 2001

26. Yelnik J, Damier P, Demeret S, Gervais D, Bardinet E, Bejjani BP, et al: Localization of stimulating electrodes in patients with Parkinson disease by using a three-dimensional atlas-magnetic resonance imaging coregistration method. J Neurosurg 99:89-99, 2003

Manuscript received April 26, 2005.

Accepted in final form March 2, 2006.

Address reprint requests to: Claudio Pollo, M.D., Department of Neurosurgery, Centre Hospitalier Universitaire Vaudois, 1011 Lausanne, Switzerland. email: Claudio.Pollo@chuv.hospvd.ch. 